\title{
Generation of broad spectral components from midwave infrared ultrashort pulse laser propagation through ZnSe and ZnS
}

Kevin Werner, Michael Tripepi, Aaron Schweinsberg, Michael Hastings, Laura Vanderhoef, et al.

Kevin Werner, Michael Tripepi, Aaron Schweinsberg, Michael Hastings, Laura Vanderhoef, Christopher Wolfe, Trenton Ensley, Brian Wilmer, Miroslav Kolesik, Jerome Moloney, Enam Chowdhury, Anthony Valenzuela, "Generation of broad spectral components from midwave infrared ultrashort pulse laser propagation through ZnSe and ZnS," Proc. SPIE 10902, Nonlinear Frequency Generation and Conversion: Materials and Devices XVIII, 109020W (4 March 2019); doi: 10.1117/12.2509928 


\title{
Generation of Broad Spectral Components from Midwave Infrared Ultrashort Pulse Laser Propagation through ZnSe and $\mathrm{ZnS}$
}

\author{
Kevin Werner $^{\mathrm{a}}$, Michael Tripepi ${ }^{\mathrm{a}}$, Aaron Schweinsberg ${ }^{\mathrm{b}}$, Michael Hastings ${ }^{\mathrm{c}}$, Laura \\ Vanderhoef $^{\mathrm{b}}$, Christopher Wolfe ${ }^{\mathrm{b}}$, Trenton Ensley ${ }^{\mathrm{d}}$, Brian Wilmer ${ }^{\mathrm{e}}$, Miroslav Kolesik ${ }^{\mathrm{c}}$, Jerome \\ Moloney $^{\mathrm{c}}$, Enam Chowdhury ${ }^{\mathrm{a}}$, and Anthony Valenzuela ${ }^{\mathrm{b}}$ \\ aThe Ohio State University, Department of Physics. 191 W. Woodruff Ave., Columbus OH \\ 43224, USA \\ ${ }^{b}$ U.S. Army Research Laboratory, Weapons and Materials Research Directorate, Aberdeen \\ Proving Ground, MD 21005-5066, USA \\ ${ }^{\mathrm{c} C}$ College of Optical Sciences, University of Arizona, 1630 E. University Blvd., Tucson AZ \\ 85721, USA \\ ${ }^{d}$ U.S. Army Research Laboratory, Sensors and Electron Devices Directorate, Adelphi, MD \\ 20783, USA \\ eSURVICE Engineering, 4695 Millennium Drive, Belcamp, Maryland 21017, USA
}

\begin{abstract}
We investigate the nonlinear optical properties of ZnSe and ZnS using ultrashort (pulse duration approximately $200 \mathrm{fs}$ ) midwave infrared laser pulses between 3 and $4 \mu \mathrm{m}$. Multiple harmonic generation in both materials was observed, as well as significant spectral modification of the fundamental pulse. Simulations using a nonlinear polarization model enhanced with ionization compared favorably with experimental data. Random quasi phase matching in the materials is the likely generator of the observed harmonics.
\end{abstract}

Keywords: Ultrashort pulse lasers, high harmonic generation, filamentation, UPPE

\section{INTRODUCTION}

Recent advances in both the generation and simulation of mid-infrared (MIR) ultrashort pulse lasers (USPL) have lead to the discovery of new physics with distinguishable phenomena compared to more traditional nearinfrared reactions. A hallmark of MIR laser pulse propagation in air is a low-loss wavelength window in the 3-4 $\mathrm{\mu m}$ band and these wavelengths are considered "retina-safe" due to opacity of the human lens limiting most damage to the cornea. ${ }^{1}$ Similarly, visible light sensors are also less susceptible to damage from a MIR source, primarily since most common glasses (e.g. UVFS, BK-7, etc.) used for optics have low transmissivity in this range. State of the art MIR USPLs are now approaching output peak powers over a $1 \mathrm{TW}\left(10^{12} \mathrm{~W}\right)$ in this band, with pulse durations on the order of 100 femtoseconds occurring as a result of increasing conversion efficiency from near-infrared (NIR) USPL seed sources. ${ }^{2,3}$ On the horizon are more capable shortwave-infrared (SWIR) USPL sources ${ }^{4}$ that may lead to a tremendous increase in MIR generation efficiency.

Some of the key features of the new MIR USPL physics includes advances in filamentation and harmonic generation. Filamentation is a unique propagation regime of USPLs in a transparent medium where an intense pulse causes Kerr lensing via the third-order nonlinear index of refraction, $n_{2}$. As the intensity increases, a defocusing phenomenon like plasma defocusing can, accounting for losses, balance against self-focusing. ${ }^{5}$ An estimate of the peak laser power needed for filamentation is known as $P_{c r}$ given by: ${ }^{6}$

Further author information: (Send correspondence to A.V.)

A.V.: E-mail: anthony.r.valenzuela6.civ@mail.mil, Telephone: 14102789876

Nonlinear Frequency Generation and Conversion: Materials and Devices XVIII, edited by

Peter G. Schunemann, Kenneth L. Schepler, Proc. of SPIE Vol. 10902, 109020W

(C) 2019 SPIE · CCC code: 0277-786X/19/\$18 - doi: 10.1117/12.2509928

Proc. of SPIE Vol. 10902 109020W-1

Downloaded From: https://www.spiedigitallibrary.org/conference-proceedings-of-spie on 23 Jul 2019 


$$
P_{c r}=\frac{3.72 \lambda^{2}}{8 \pi n_{0} n_{2}}
$$

where $\lambda$ is the laser wavelength, and $n_{0}$ is the linear index of refraction. It is important to note that most transparent materials have $n_{0}$ within a range of approximately 1-3. However, values for $n_{2}-$ in units of $\mathrm{cm}^{2} / \mathrm{W}-$ may have a wider range even by orders of magnitude between gases and solids. Another key feature of filamentation is the generation of broadband supercontinuum peaked at the fundamental but often extending across many spectral bands depending on the linear transmissivity of the media. ${ }^{7,8}$

A majority of the filamentation body of work uses NIR, but with the increasing capabilities of MIR USPLs, new research is highlighting some of the differences at these longer wavelengths. ${ }^{9}$ From Eq. 1, we note that $P_{c r}$ is proportional to $\lambda^{2}$, meaning that all else being equal, it would take almost 10 times as much peak power to self-focus at $3.1 \mathrm{\mu m}$ as compared to $1 \mathrm{\mu m}$. High harmonic generation (HHG) has taken a significant step towards coherent x-rays by using MIR USPL filamentation in noble gases. ${ }^{10}$ The longer wavelength allows for more efficient driving of bound electrons leading to higher output of coherent x-rays beyond $1 \mathrm{keV} .{ }^{11}$ Further interest in broadening HHG further into the x-ray regime has been a significant driver to develop more capable MIR USPLs as well as extending the theoretical models to longer wavelengths. ${ }^{12}$

Expanding upon this new knowledge regarding MIR propagation in gasses, transparent solids offer another area ripe for examination. Preliminary results have already demonstrated unusual spectral features from MIR interactions with fluoride glasses ${ }^{13}$ that are not readily seen with NIR drivers. We seek to expand upon fluoride glasses by examining the effects of MIR USPLs on other common visible, NIR, SWIR, and MIR transparent optical materials. In particular, we focus on single-crystal and poly-crystal ZnSe and a common form of ZnS (hot isostatically pressed), Cleartran ${ }^{\mathrm{TM}}$. Our previous work ${ }^{14}$ with ZnSe proved not only that we are able to generate odd and even order harmonics but also provide good qualitative agreement with simulations; we continue this effort with Cleartran. Reviewing Eq. 1, we not only have to consider a quadratic increase with wavelength, but we also have to be cautious regarding the values we choose for $n_{2}$ given that most of the data has been limited to NIR. To that end, we include z-scan measurements ${ }^{15}$ of our materials to provide $n_{2}$ at relevant MIR wavelengths and intensities in the range of our experiments. This also allows us to model these interactions with a higher degree of certainty.

\section{EXPERIMENTAL SETUP}

Our work employed two USPLs to perform the experimental component, one at The Ohio State University (OSU) and one at the US Army Research Laboratory (ARL-ALC). The OSU laser is the Extreme MIR (EMIR) optical parametric amplifier (OPA) pumped by the $500 \mathrm{~Hz}$ repetition rate Ti:Sapphire OSU Gray Laser as shown in Fig. 1a. The output of EMIR is continuously tunable between 2.5 and $4.5 \mu \mathrm{m}$ with a pulse duration of $200 \mathrm{fs}$ at a $500 \mathrm{~Hz}$ repetition rate. For the scope of our experiments, we utilized central fundamental wavelengths between 3 and $4 \mu \mathrm{m}$ in keeping with the atmospheric propagation window in the MIR. In addition, we took advantage of the zero dispersion point of $\mathrm{ZnS}$ at $3.6 \mu \mathrm{m}$. Energy tuning of our pulse between 1 and $10 \mu \mathrm{J}$ is accomplished using a waveplate/polarizer system. This translates to peak powers ranging from 5 to $50 \mathrm{MW}$, well below the $P_{c r}$ in air of $250 \mathrm{GW}$ (for $3.6 \mu \mathrm{m}$ ). The pulses were focused onto the sample using $\mathrm{CaF}_{2}$ lenses with focal lengths varying from 20 to $105 \mathrm{~mm}$, achieving spot sizes ranging from 24 to $98.5 \mu \mathrm{m}$ FWHM for 20 and $105 \mathrm{~mm}$ lenses, respectively. Thus, we achieve focal intensities in the range of $0.06-11 \mathrm{TW} / \mathrm{cm}^{2}$. A list of the samples used in this experiment is given in Table 1. To compensate for losses in transmissive optics, energy readings for the light focused into the samples were taken at point $E_{1}$ as shown in Fig. 1a. The emission from the sample is sent into either a Vis/NIR channel or a MIR channel by using a flippable mirror. The Vis/NIR emission was collected with a microscope objective that filtered out the MIR signal and fed into a fiber connected to to a suite of spectrometers covering 0.4 to $2.5 \mu \mathrm{m}$. We also were able to take energy measurements of the Vis/NIR signal by placing an energy meter at position $E_{2}$. The MIR channel focused the emission onto either a MIR spectrometer or camera. The MIR spectrometer was from Acton Research with a FLIR cooled focal plane array capable of detecting 3-5 $\mu \mathrm{m}$ light. 


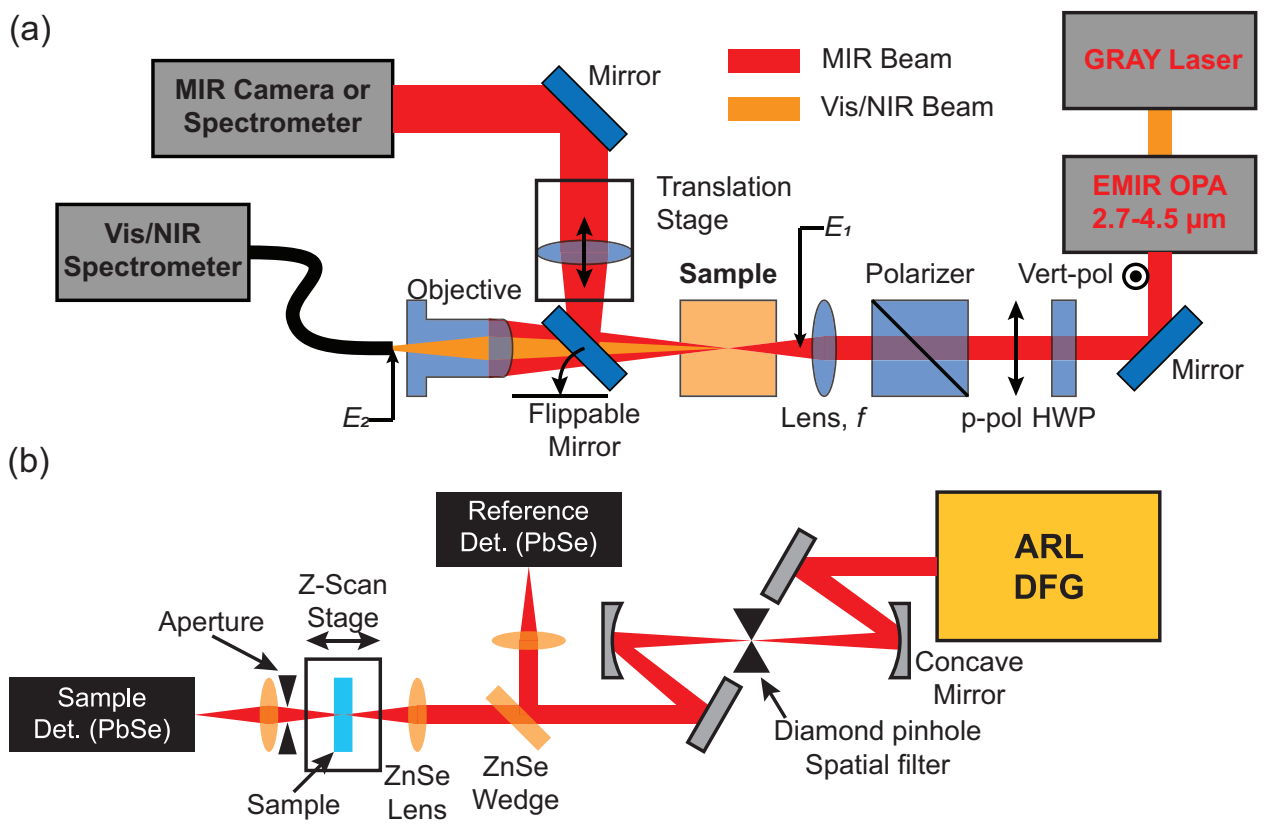

Figure 1. Experimental setup: (a) OSU's EMIR OPA for generating MIR laser pulses. MIR pulse energy is controlled using a half waveplate (HWP) polarizer combination. MIR light is focused by a lens $f$ onto a sample. The emission from the sample is collected either with a microscope objective that filters out the MIR spectrum and focuses the light into a fiber attached to a Vis or NIR spectrometer or a $\mathrm{CaF}_{2}$ lens that collimates the light into a MIR camera or spectrometer (flippable mirror controls which detection line is used). For harmonic efficiency measurements, pulse energy was measured at $E_{1}$ and $E_{2}$. (b) ARL-ALC setup of non-linear index, $n_{2}$ measurement with MIR femtosecond pulses using Z-scan technique using reflective geometry.

Table 1. List of samples used for experiments including relevant parameters.

\begin{tabular}{|l|l|l|l|l|l|}
\hline Name & Material & Type & Structure (grain size in $\mu \mathrm{m})$ & Thickness $(\mathrm{mm})$ & Bandgap $(\mathrm{eV})$ \\
\hline poly-ZnSe & ZnSe & Polycrystalline & Cubic $(20-100)$ & $1,5,10,20,40$ & 2.82 \\
\hline single-ZnSe & ZnSe & Single Crystal & Cubic & 1 & 2.82 \\
\hline Cleartran & ZnS & Polycrystalline & Cubic $(20-35)$ & $3,10,20,40$ & 3.54 \\
\hline
\end{tabular}

The ARL-ALC experimental setup as shown in Fig. $1 \mathrm{~b}$ was used for characterization of $n_{2}$ for our samples using the Z-scan technique. MIR pulses at $3.9 \mu \mathrm{m}$ were generated by using a Ti:sapphire laser system (Coherent, Hidra-F-100) to pump an OPA (Light Conversion, TOPAS-Prime-HE), which was then used for difference frequency generation, DFG (2 mm thick Type II KTA). The DFG pulses were spatially filtered by focusing into a $180 \mu \mathrm{m}$ diameter single-diamond pinhole using an all-reflective geometry. A PbSe reference detector used the pickoff from a ZnSe wedge. The remaining light was focused into the sample using a $150 \mathrm{~mm} \mathrm{ZnSe}$ focusing lens with the transmitted light from the sample focused onto a second PbSe detector. Energy readings in the Z-scan system were accomplished using a spectrally flat broadband $\mathrm{THz}$ radiometer with linearity ranging from $0.1 \mu \mathrm{W}$ to $20 \mathrm{~mW}$ calibrated with a pyroelectric detector near the DFG output. The radiometer was then placed just before the sample without any filtering to record the actual energy. Germanium neutral density filters were used to attenuate the energy used in the Z-scan runs. The beam size was measured to be $48 \mu \mathrm{m}$ gaussian waist radius using a knife-edge scan and the pulse duration was measured to be $260 \mathrm{fs}$ (FWHM) using second-order autocorrelation. 


\section{NUMERICAL MODELING}

Our simulation of MIR USPL interactions with ZnSe and ZnS utilized the gUPPEcore simulator implemention of the unidirectional pulse propagation equations (UPPE). ${ }^{16}$ The advantage of using UPPE to model this interaction is the preservation of nonlinear responses in polarization and current density that are critical to succesfully capture spectral evolution. Nonlinear polarization incorporates both second-order and third-order nonlinearities, with the former modeled by an effective isotropic medium where

$$
P_{2}(t)=r(z) \epsilon_{0} d_{e f f} E^{2}(t)
$$

where $r(z)$ is a random function for propagation through polycrystalline grains and $d_{\text {eff }}$ is the effective nonlinear coefficient. To model the effect of random grains in poly-ZnSe and Cleartran, $r(z)$ is turned on and off with a frequency in the range of average grain size. For poly-ZnSe we use $d_{\text {eff }}=15 \mathrm{pm} / \mathrm{V}$ and grain size of $70 \mu \mathrm{m}$. For comparison, poly-ZnSe has grain sizes ranging from 20 to $100 \mu \mathrm{m}$ and $L_{c o h} \sim 51,17$, and $9.6 \mu \mathrm{m}$ for the $2^{\text {nd }}$, $3^{\text {rd }}$, and $4^{\text {th }}$ harmonics via $\chi^{(2)}$ nonlinearity, respectively. The third-order nonlinearity depends on experimental values from our Z-scan effort. Model components like carrier generation, chromatic dispersion, and linear optical losses utilize measured parameters, leaving $d_{e f f}$ as the only adjustable parameter.

The simplified model developed here allows for manageable computation complexity owing to the need to gain statistical understanding when comparing against natural variability expected from polycrystalline data. To further simplify the modeling, we use radial symmetry with a single filament propagation. By averaging over multiple runs with random orientation and size of grains with very fine spatial resolution, we converge upon a reasonable simulation of the interaction. However, this limits us to a qualitative comparison with the data with a quantitative comparison likely requiring the ability to simulate multiple filaments.

As a next step toward a comprehensive treatment of poly-crystalline nonlinear media, we present preliminary results for an improved model. In contrast to the effective approach discussed above, we honor the vector nature of the electric field, and describe the second-order nonlinearity with the proper $\chi^{(2)}$ tensor. In order to keep the computational complexity in check, we still assume that the focused laser beam diameter fits (transversely) into a single crystallite. Each crystallite has a random orientation, which changes after a characteristic "correlation length," thus mediating the disorder that is necessary for the random quasi-phase matching.

The main advantage of this model lies in the fact that it can be applied to both single- and poly-crystalline media, and the nonlinear parameter $d_{\text {eff }}$ is now a microscopic quantity rather than an effective-model parameter. Figure 2 shows simulated spectra for a $5 \mathrm{~mm}$ sample of poly-crystalline ZnSe with $d_{\text {eff }}=30 \mathrm{pm} / \mathrm{V}$. The top panel illustrates the spectrum in its entirety, revealing that with the exception of the fundamental-frequency band the radiation becomes depolarized even after a few millimeters of propagation through the random medium. The bottom panel shows a zoomed in view of the portion of the spectra that should be compared with the experimental data depicted in Fig 5b. It is encouraging that this improved model shows significantly better agreement with the measurement, where in particular the high-harmonic bands attain powers quite similar to those measured.

\section{RESULTS AND DISCUSSION}

The ARL-ALC setup allowed us to measure the absolute $n_{2}$ values of ZnSe and Cleartran at MIR wavelengths using the Z-scan technique. For poly-ZnSe, a $2 \mathrm{~mm}$ thick sample was used with a MIR wavelength of $3.9 \mathrm{\mu m}$ and focal intensities from 4.7 to $19 \mathrm{GW} / \mathrm{cm}^{2}$ to arrive at an average $n_{2}$ of $(1.2 \pm 0.3) \times 10^{-14} \mathrm{~cm}^{2} / \mathrm{W}$. This value compares favorably with the theoretical prediction from Eq. 44 in Ref. 17 given a bandgap energy, $E_{g}=2.71 \mathrm{eV}$, $\mathrm{K}=3100, \mathrm{~K}^{\prime}=1.5 \times 10^{-8}$, and $E_{p}=21.4 \mathrm{eV}$. Similarly, Z-scan measurements were made with a $3 \mathrm{~mm}$ Cleartran sample with 4 laser energies at $3.9 \mu \mathrm{m}$ and an average $n_{2}$ was measured to be $(5.0 \pm 1.3) \times 10^{-15} \mathrm{~cm}^{2} / \mathrm{W}$. From these results we can then use Eq. 1 to compute $P_{c r}$ for our samples as shown in Table 2. Thus with 5-50 MW peak power pulses we are well into the multiple filamentation regime in our ZnSe and Cleartran samples, though there is significant complexity regarding the length of the sample that is not captured in Eq. 1.

The expeirments conducted at OSU (see Fig. 1b) with ZnSe and Cleartran produced emission in the visible to the MIR bands. We can see from Fig. 3 that a change in energy of the pulse results in broadening of the fundamental pulse for even a thin ZnSe sample. This is due to self-phase modulation of the beam and multiple 

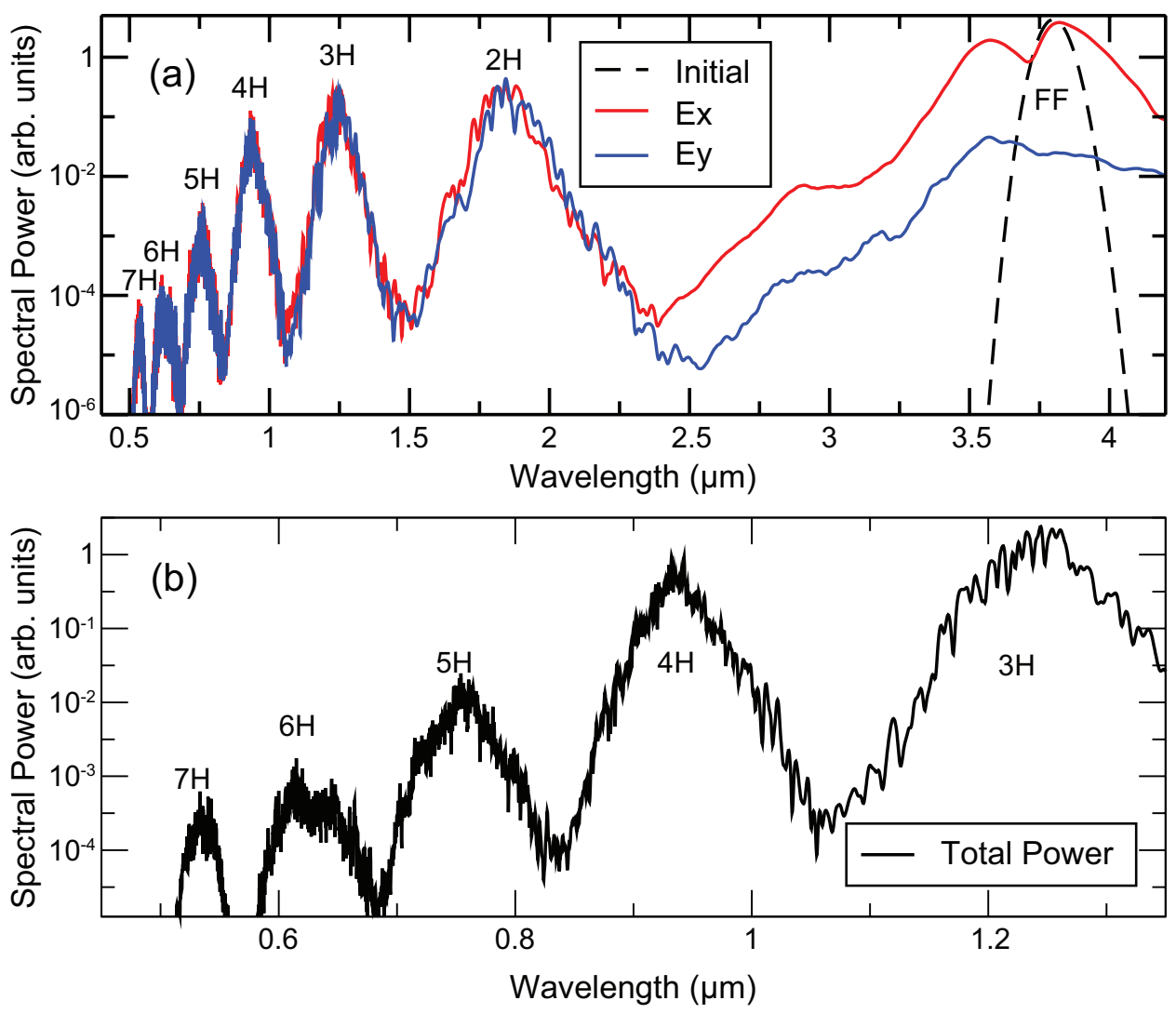

Figure 2. Simulated spectra for $5 \mathrm{~mm}$ poly-ZnSe sample using vectorial model with randomly oriented crystallites. a) Initially linearly polarized pump (dashed) gives rise to several broadened harmonic orders in which the original (Ex) and perpendicular (Ey) polarizations carry essentially the same power below $2.4 \mu \mathrm{m}$. b) Detail of the unpolarized harmonic spectra showing non-perturbative behavior with a very slow decrease of the spectral power with increasing harmonic order.
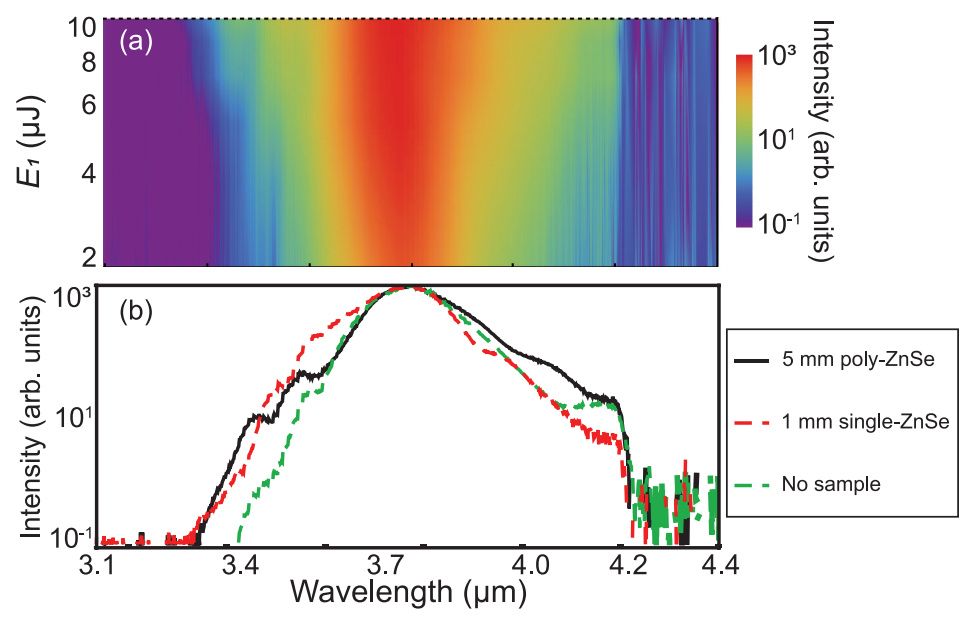

Figure 3. Experimental measurements from Ref. 14 of transmitted fundamental spectral intensity (a) mapped as a function of MIR pulse (center wavelength $\lambda=3.8 \mu \mathrm{m}$ ) energy focused onto a $5 \mathrm{~mm}$ thick poly-ZnSe. Linear interpolation is used for ease of reading. The dotted black line at the top boundary represents a lineout presented in (b) (black curve), for an input MIR pulse energy $\left(E_{1}=10.6 \mathrm{\mu J}\right)$. (b) Comparing MIR spectral broadening in $5 \mathrm{~mm}$ poly-ZnSe and $1 \mathrm{~mm}$ single-ZnSe data (red dotted line, $E_{1}=10.4 \mathrm{\mu J}$ ), along with that of the laser without any sample (green dotted line). 


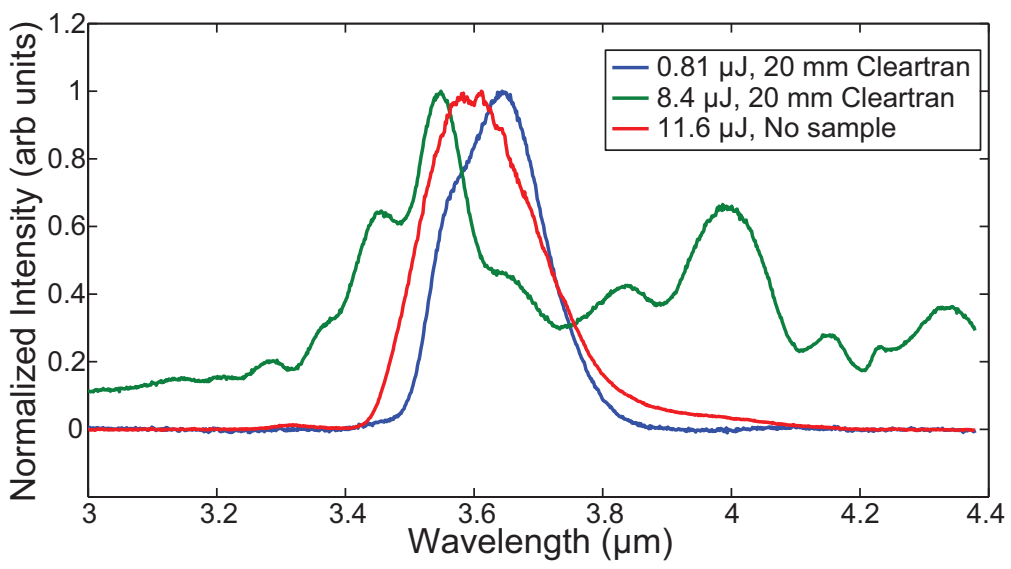

Figure 4. Comparison of MIR spectral data with a $3.6 \mu \mathrm{m}$ fundamental between the unperturbed spectra and emission from $20 \mathrm{~mm}$ of Cleartran at $0.81 \mu \mathrm{J}$ and $8.4 \mu \mathrm{J}$. Note linear scale of intensity.

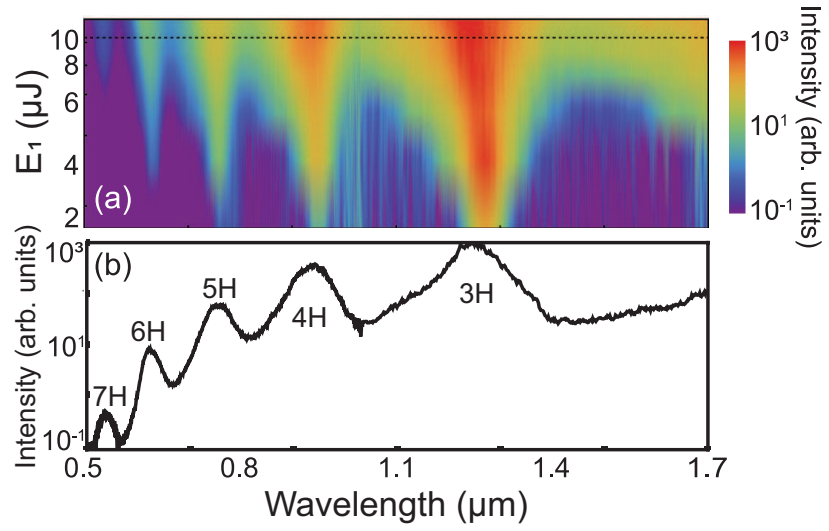

Figure 5. Experimental spectral measurements from Ref. 14 of transmitted generated harmonics intensity as a function of MIR pulse energy in a $5 \mathrm{~mm}$ thick poly-ZnSe sample. (a) Spectral mapping for harmonics resulting from $\lambda=3.8$ pm MIR driver laser. Linear interpolation is used for ease of reading. The dotted black line in (a) represents a lineout presented in (b) (black curve), for an input MIR pulse energy of $E_{1}=10.6 \mu \mathrm{J}$.

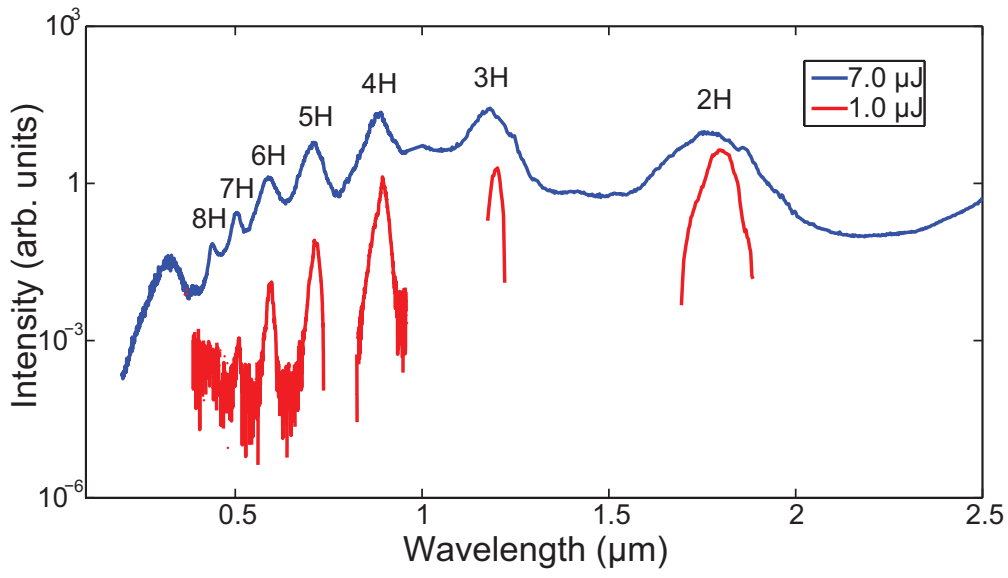

Figure 6. Visibile, NIR, and SWIR emission spectra from $3 \mathrm{~mm}$ of Cleartran at $1.0 \mathrm{\mu J}$ and $7.0 \mathrm{\mu J}$ with a fundamental of $3.6 \mathrm{~mm}$. Note the lack of IHC at the lower energy level leading to isolated harmonic peaks depending on the sensitivity of the spectrometer for each wavelength region. 
filamentation. Please note that there is an artificial cutoff of the higher wavelength side of the broadened pulse beyond $4.2 \mu \mathrm{m}$ due to strong attenuation of the signal in spectral intensity from $\mathrm{CO}_{2}$ absorption. Similar broadening is seen with Cleartran in Fig. 4, however it is much more exaggerated at higher energies leading to a complex multi-peak structure.

However, we see a much more pronounced effect looking at the vis/NIR spectrum in Fig. 5 where even at lower energies there are clearly even and odd order harmonics being generated (3rd, 4th, and 5th). With increasing energy, we detect the addition of the 6th and 7th harmonics as shown in Fig. 5(b). Similar distinct harmonics are also generated from Cleartran as seen in Fig. 6. Note that Cleartran has higher linear transparency between 350 and $550 \mathrm{~nm}$ compared to ZnSe. This data strongly corroborates the hypothesis of random quasi phase matching $(\mathrm{RQPM})^{18-21}$ found in such isotropic polycrystalline materials. RQPM arises from a phase mismatch from the mixing of 3 waves of photons in a medium with non-zero $\chi^{(2)}$ such that $\Delta k=k_{3}-k_{2}-k_{1}$. The phase mismatch also determines the the coherence length, $L_{c o h}$, such that $L_{c o h}=\pi / \Delta k$. As the pulse propagates through periodically poled crystal, each $L_{c o h}$ step, $N$, causes a reversal of the wavemixing between the 3 waves leading to nonlinear field gain $\propto N$. However, both ZnSe and Cleartran are not periodically poled, yet the polycrystalline nature of these materials leads to a reversal between domain that results in a gain $\propto \sqrt{N}$ where $N$ is equivalent to the number of domains traversed. To reach an approximation of $N$ we consider the average grain size from the distribution in Table 1 and the thickness of the sample. However, thicker samples, particularly for ZnSe, will also increase the amount of linear absorption at higher harmonics reducing some amount of this gain.

By comparing energy readings at $E_{1}$ and $E_{2}$, we can measure the efficiency of harmonic generation as shown in Fig. 7 for ZnSe. We are able to achieve a high conversion efficiency of over 30\%, however it appears there are both a linear dependence on sample thickness and a saturation effect at higher energy levels that remain to be explored further. We see similar conversion efficiency for Cleartran at $10.5 \mu \mathrm{J}$ of about $30 \%$. We are unable to measure an efficiency for single-ZnSe since transmitted energies at $E_{2}$ are below our detector's threshold.

Simulations were performed for poly-ZnSe and single-ZnSe to test the capability of gUPPE to provide qualitative agreement with the experimental data. To accomplish this, simulations were perform with $\lambda=3.8 \mu \mathrm{m}$ with focal geometry matching the OSU experiment. To determine a valid $d_{\text {eff }}$, we examined the range reported in the literature from 16 to $132 \mathrm{pm} / \mathrm{V}^{22,23}$ and chose a value (15 pm/V) just below the low end that converged upon a result closer to the experimental data. Pulse energies were varied from 0.8 to $8.6 \mu \mathrm{J}$. Variations arising from the random function $r(z)$ were averaged over four realizations. The representative results are shown in Fig. 8, demonstrating specific harmonics along with an inter-harmonic continuum (IHC) that grows with increased energy. Distinctive harmonics are readily generated within $5 \mathrm{~mm}$ of propagation in poly-ZnSe at low energies, but with increased propagation distance and energies the harmonic peaks lose some distinction due to increased IHC. It is important to note the qualitative similarities between the simulations in Figs. 2 and 8 and experimental data in Fig. 5 demonstrating a remarkable similarity even for modeling a single filament.

Our simulations also modeled the evolution of the fundamental pulse in the MIR. As seen in the simulated spectra in Fig. 9 there is significant broadening on both the red and blue sides of the fundamental. This broadening is due to self-phase modulation of the pulse with an added effect of a carrier-induced blue shift from free-carrier generation, leading to an assymmetry favoring the blue side of the pulse. This part of the simulation does not readily agree with that seen from the experimental data in Fig. 3. Simulations of Cleartran are on-going and are expected to provide further evidence of the ability of gUPPE to simulate these interactions particularly in the case of zero dispersion at $3.6 \mu \mathrm{m}$.

\section{CONCLUSION AND FUTURE WORK}

Our work demonstrates novel spectral results from the interaction between MIR USPLs and optical materials that can be successfully simulated using UPPE. RQPM is the dominant mechanism that produces the harmonics we detect up to the $9^{\text {th }}$ harmonic in Cleartran and this was successfully incorporated into UPPE. Understanding how MIR light interacts with these optical materials may lead to new applications related to SWIR and MIR laser media such as Fe:ZnSe ${ }^{28}$ and $\mathrm{Cr}: \mathrm{ZnSe}^{29}$ In addition, the successful simulation results demonstrate the adaptability of UPPE to model a wide variety of transparent materials and incorporate experimentally derived values. Our future work will include simulations of Cleartran and single crystal $\mathrm{ZnS}$ as well as experimental 
Table 2. Comparison of self-focusing critical power thresholds for ZnSe, ZnS, and $\mathrm{N}_{2}$ in the NIR and MIR using the average value of $n_{2}$ and assuming minimal nonlinear dispersion. Values for $n_{0}$ and $n_{2}$ were obtained from the references listed below except for $\mathrm{ZnS}$ where $n_{2, \mathrm{NIR}}$ was previously measured by T. Ensley and $n_{2, \mathrm{MIR}}$ that was measured as part of this work.

\begin{tabular}{|l|c|c|c|c|l|}
\hline \multirow{2}{*}{ Material } & \multicolumn{4}{|c|}{$P_{c r}(\mathrm{MW})$} & \multirow{2}{*}{ Refs. } \\
\cline { 2 - 5 } & $\lambda=0.8 \mu \mathrm{m}$ & $\lambda=3.0 \mu \mathrm{m}$ & $\lambda=3.6 \mu \mathrm{m}$ & $\lambda=3.8 \mu \mathrm{m}$ & \\
\hline $\mathrm{ZnSe}$ & 0.01 & 0.46 & 0.66 & 0.74 & $24,25,14$ \\
\hline $\mathrm{ZnS}$ & 0.07 & 1.2 & 1.7 & 1.9 & 24 \\
\hline $\mathrm{N}_{2}$ & $1.3 \times 10^{5}$ & $1.8 \times 10^{5}$ & $2.5 \times 10^{5}$ & $2.8 \times 10^{5}$ & $24,26,27$ \\
\hline
\end{tabular}

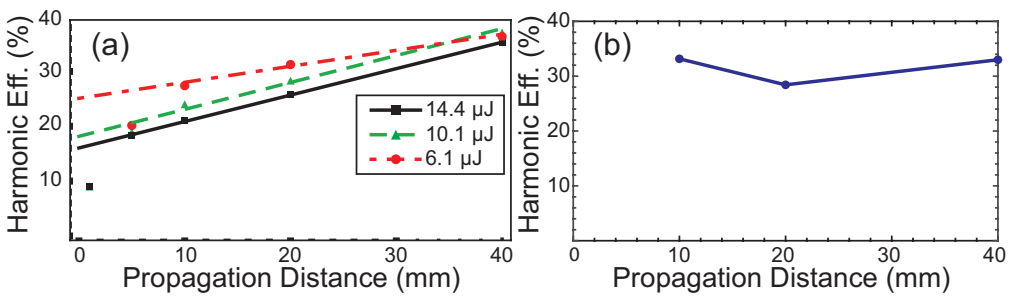

Figure 7. Harmonic conversion efficiency for (a) ZnSe depending on thickness of sample and pulse energy for $\lambda=3.3 \mu \mathrm{m}$ (from Ref. 14) and (b) Cleartran depending on sample thickness at $E_{1}=10.6 \mu \mathrm{J}$ and $\lambda=3.6 \mu \mathrm{m}$.
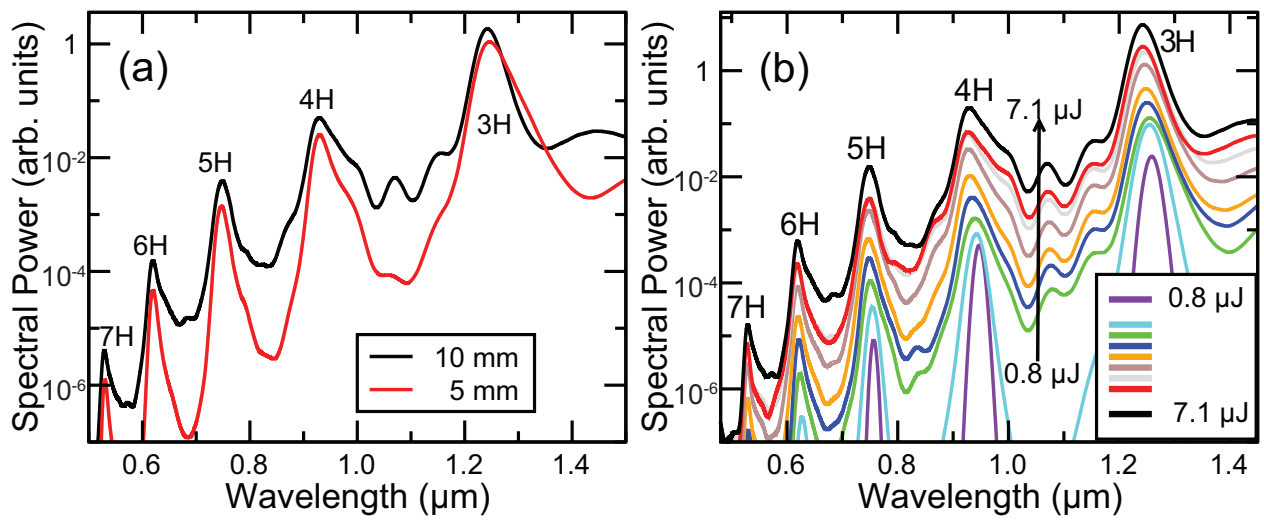

Figure 8. Simulated spectra from Ref. 14 for poly-ZnSe with (a) spectra at two different propagation distances in the sample for a pulse with the initial energy of $7.1 \mu \mathrm{J}$. (b) Spectra at propagation distance $z=10 \mathrm{~mm}$ for a range of pulse energies between 0.8-7.1 $\mathrm{\mu J}$. 


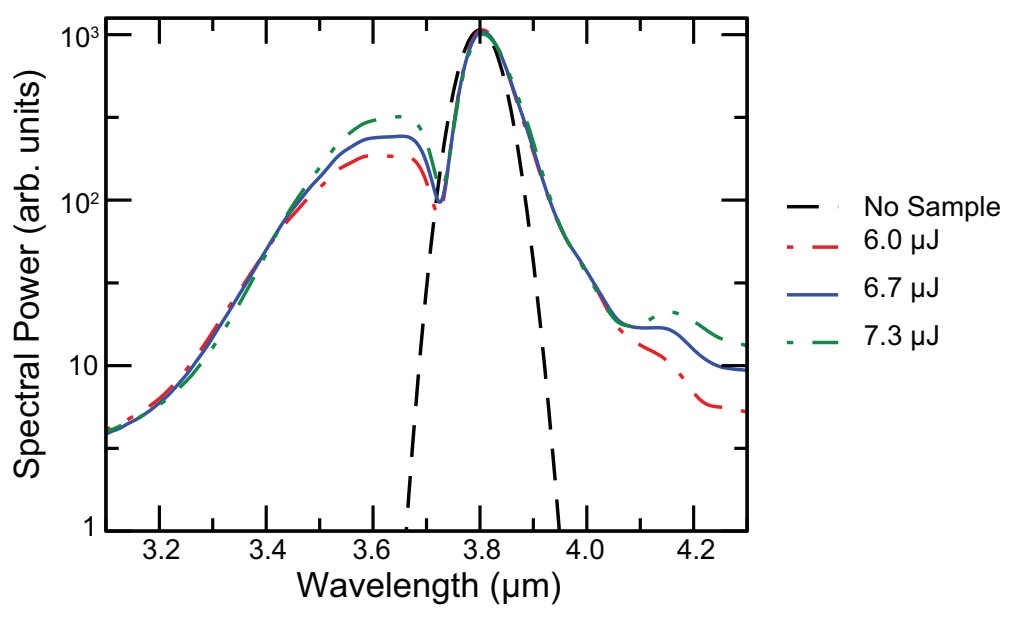

Figure 9. Simulated spectra from Ref. 14 for poly-ZnSe demonstrating broadening of the fundamental at $\lambda=3.8 \mu \mathrm{m}$.
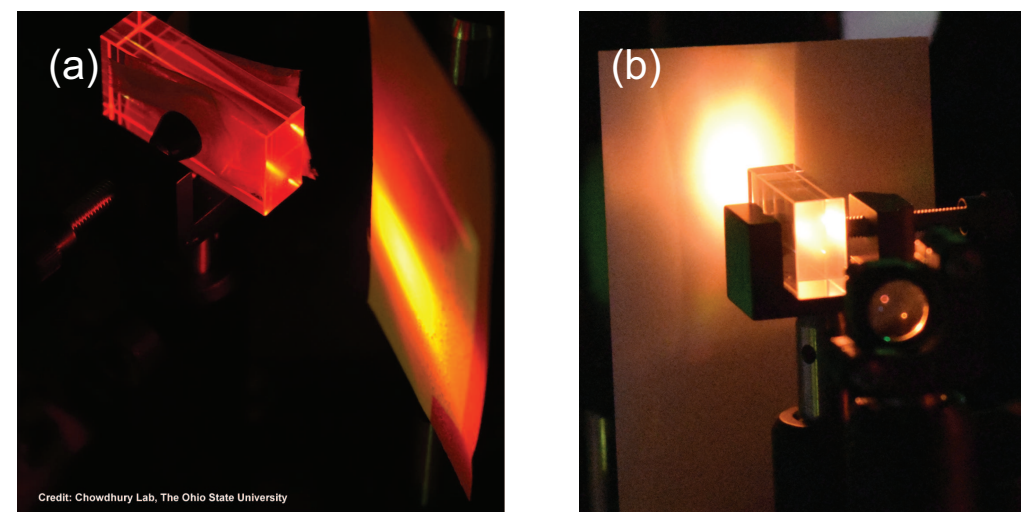

Figure 10. Photographs of $40 \mathrm{~mm}$ samples and resultant visible emission from (a) poly-ZnSe (from Ref. 14) and (b) Cleartran. Note the MIR USPL propagates from the top left in (a) and bottom right in (b). 
studies of the latter. In addition, we can compare the conversion efficiency into harmonics and IHC with supercontinuum generation. Further development of UPPE will seek to have a better understanding of SPM's role in the broadening of the fundamental. We will also continue to perform nonlinear index of refraction measurements of a wide variety of optical materials in the MIR. Interest in MIR is being joined by increased interest in longwave infrared $(8-20 \mu \mathrm{m})$ which may lead to further understanding of harmonic generation and efficient conversion into visible to MIR wavelengths.

\section{ACKNOWLEDGMENTS}

The authors would like to acknowlesge support from SURVICE (S17-095008/DOTC-17-01-INIT0086); Air Force Office of Scientific Research (AFOSR) (FA9550-16-1-0069); AFOSR multidisciplinary research program of the university research initiative (MURI) (FA9550-16-1-0013); AFOSR (FA9550-16-1-0121). The authors would also like to thank StingRay Optics for their assistance.

\section{REFERENCES}

[1] Zuclich, J. A., Lund, D. J., and Stuck, B. E., "Wavelength dependence of ocular damage thresholds in the near-IR to far-IR transition region: proposed revisions to MPEs," Health Phys. 92, 15-23 (2007).

[2] Mitrofanov, A. V., Voronin, A. A., Sidorov-Biryukov, D. A., Pugžlys, A., Stepanov, E. A., Andriukaitis, G., Flöry, T., Ališauskas, S., Fedotov, A. B., Baltuška, A., and Zheltikov, A. M., "Mid-infrared laser filaments in the atmosphere," Sci. Rep. 5, 8368 (2015).

[3] Pires, H., Baudisch, M., Sanchez, D., Hemmer, M., and Biegert, J., "Ultrashort pulse generation in the mid-IR," Prog. Quantum Electron. 43, 1-30 (2015).

[4] Cole, B., Goldberg, L., Chinn, S., Pomeranz, L. A., Zawilski, K. T., Schunemann, P. G., and McCarthy, J., "Compact and efficient mid-IR OPO source pumped by a passively Q-switched Tm:YAP laser," Opt. Lett. 43, 1099-1102 (2018).

[5] Couairon, A. and Mysyrowicz, A., "Femtosecond filamentation in transparent media," Phys. Rep. 441, 47-189 (2007).

[6] Marburger, J. H., "Self-focusing: Theory," Prog. Quant. Electr. 4, 35-110 (1975).

[7] Kasparian, J., Sauerbrey, R., Mondelain, D., Niedermeier, S., Yu, J., Wolf, J.-P., André, Y.-B., Franco, M., Prade, B., Tzortzakis, S., Mysyrowicz, A., Rodriguez, M., Wille, H., and Wöste, L., "Infrared extension of the supercontinuum generated by femtosecond terawatt laser pulses propagating in the atmosphere," Opt. Lett. 25, 1397-1399 (2000).

[8] Dubietis, A., Tamošauskas, G., Šuminas, R., Jukna, V., and Couairon, A., "Ultrafast supercontinuum generation in bulk condensed media," Lith. J. Phys. 57, 113-157 (2017).

[9] Zheltikov, A. M., "Laser-induced filaments in the mid-infrared," J. Phys. B 50, 092001 (2017).

[10] Hernández-García, C., Pérez-Hernández, J. A., Popmintchev, T., Murnane, M. M., Kapteyn, H. C., JaronBecker, A., Becker, A., and Plaja, L., "Zeptosecond high harmonic keV x-ray waveforms driven by midinfrared laser pulses," Phys. Rev. Lett. 111, 033002 (2013).

[11] Emelina, A. S., Emelin, M. Y., and Ryabikin, M. Y., "Multi-keV ultrahigh-order harmonics produced in gases with subrelativistically intense mid-IR laser pulses," J. Opt. Soc. Am. B 32, 2478-2487 (2015).

[12] Emelin, M. and Ryabikin, M., "On the potential of mid-IR lasers for generating high harmonics with subnanometer wavelengths in gases," Quant. Electron. 43, 211-216 (2013).

[13] Dormidonov, A., Kompanets, V., Chekalin, S., and Kandidov, V., "Giantically blue-shifted visible light in femtosecond mid-IR filament in fluorides," Opt. Express 23, 29202-29210 (2015).

[14] Werner, K., Hastings, M. G., Schweinsberg, A., Wilmer, B. L., Austin, D., Wolfe, C. M., Kolesik, M., Ensley, T. R., Vanderhoef, L., Valenzuela, A., and Chowdhury, E., "Ultrafast mid-infrared high harmonic and supercontinuum generation with $n_{2}$ characterization in zinc selenide," Opt. Express accepted (2019).

[15] Sheik-Bahae, M., Said, A., Wei, T.-H., Hagan, D., and Stryland, E. V., "Sensitive measurement of optical nonlinearities using a single beam," IEEE J. Quant. Electr. 26, 760-769 (1990).

[16] Kolesik, M. and Moloney, J. V., "Nonlinear optical pulse propagation simulation: from Maxwell's to unidirectional equations," Phys. Rev. E 70, 036604 (2004). 
[17] Sheikbahae, M., Hutchings, D. C., Hagan, D. J., and Vanstryland, E. W., "Dispersion of bound electronic nonlinear refraction in solids," IEEE J. Quant. Electron. 27, 1296-1309 (1991).

[18] Morozov, E. Y., Kaminskii, A. A., Chirkin, A. S., and Yusupov, D. B., "Second optical harmonic generation in nonlinear crystals with a disordered domain structure," JETP Lett. 73, 647-650 (2001).

[19] Chinh, T. D., Seibt, W., and Siegbahn, K., "Dot patterns from second-harmonic and sum-frequency generation in polycrystalline ZnSe," J. Appl. Phys. 90, 2612-2614 (2001).

[20] Baudrier-Raybaut, M., Haidar, R., Kupecek, P., Lemasson, P., and Rosencher, E., "Random quasi-phasematching in bulk polycrystalline isotropic nonlinear materials," Nature 432, 374-376 (2004).

[21] Skipetrov, S. E., "Nonlinear optics - disorder is the new order," Nature 432, 285-286 (2004).

[22] Weber, M. J., [CRC handbook of laser science and technology], CRC Press, New York (1986 (third edition)).

[23] Piltch, M. S., Eckhardt, R., and Hinsley, R., "Determination of the nonlinear optical coefficient and SHG coherence length for crystal single zinc selenide," Opt. Commun. 22, 239-242 (1977).

[24] Querry, M., "Optical constants of minerals and other materials from the millimeter to the ultraviolet," Contractor Report, CRDEC, CR-88009 (1987).

[25] Tseng, K. Y., Wong, K. S., and Wong, G. K. L., "Femtosecond time-resolved z-scan investigations of optical nonlinearities in ZnSe," Opt. Lett. 21, 180-182 (1996).

[26] Wahlstrand, J. K., Cheng, Y.-H., and Milchberg, H. M., "Absolute measurement of the transient optical nonlinearity in $\mathrm{N}_{2}, \mathrm{O}_{2}, \mathrm{~N}_{2} \mathrm{O}$, and Ar," Phys. Rev. A 85, 043820 (2012).

[27] Zahedpour, S., Wahlstrand, J. K., and Milchberg, H. M., "Measurement of the nonlinear refractive index of air constituents at mid-infrared wavelengths," Opt. Lett. 40, 5794-5797 (2015).

[28] Dai, S., Feng, G., Zhang, H., Ning, S., Xiao, Y., and Zhou, S., "4.24 $\mu \mathrm{m}$ mid-infrared laser based on a single $\mathrm{Fe}^{2+}$-doped ZnSe microcrystal," Opt. Lett. 43, 411-414 (2018).

[29] Wang, Y., Fernandez, T. T., Coluccelli, N., Gambetta, A., Laporta, P., and Galzerano, G., "47-fs Kerr-lens mode-locked Cr:ZnSe laser with high spectral purity," Opt. Express 25, 25193-25200 (2017). 